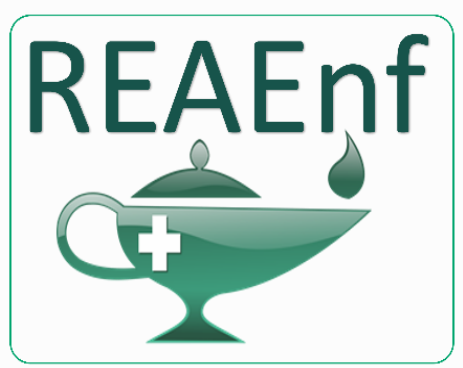

Revista Eletrônica Acervo Enfermagem
REVISÃO BIBLIOGRÁFICA

Recebido em: 8/2020

Aceito em: 9/2020

Publicado em: 11/2020

\title{
Benefícios do tratamento com oxigenoterapia hiperbárica em úlcera venosa
}

\author{
Benefits of hyperbaric oxygenotherapy in the treatment of venous ulcer
}

Beneficios del tratamiento con oxigenoterapia hiperbárica en la úlcera venosa

Laysa de Oliveira Lima ${ }^{1}$, Izabela Leopoldina da Paz Alcantara Marques ${ }^{1}$, Laís Alves Guerra Nascimento ${ }^{1}$, Jacqueline da Silva Nascimento ${ }^{1}$, Renata da Silva Schulz ${ }^{2 \star}$, Rose Ana Rios David ${ }^{2}$, Darci de Oliveira Santa Rosa².

Resumo: Esse artigo buscou verificar, na literatura, os benefícios da oxigenoterapia hiperbárica em pessoas com úlcera venosa. Trata-se de revisão integrativa realizada nas bases de dados eletrônicas Biblioteca Virtual de Saúde (BVS), National Library of Medicine (PubMed), e Web of Science. Utilizou-se os descritores em português e inglês: Úlcera Varicosa AND Oxigenoterapia. A associação dos descritores possibilitou a seleção de quarenta (40) artigos sem inclusão de limite temporal, cinco (05) artigos foram incluídos na revisão. A oxigenoterapia hiperbárica apresentou efeito de alto potencial de cicatrização em feridas venosas crônicas. Os efeitos demonstrados foram: melhora da hipóxia tecidual, aumento da perfusão, redução do edema, queda na regulação das citocinas inflamatórias, proliferação de fibroblastos, produção de colágeno e angiogênese. A revisão demonstrou eficácia no tratamento da úlcera venosa complexa com a oxigenação hiperbárica, pois houve redução dos scores de dor, aumento do processo cicatricial e de cura, porém não foi possível observar efeitos em úlceras não complexas.

Palavras-chave: Úlcera venosa, Oxigenoterapia, Cicatrização.

\begin{abstract}
This article aims to verify, in the literature, the benefits of hyperbaric oxygen therapy in people with venous ulcers. The methodology is an integrative review conducted in the Virtual Health Library (BVS), National Library of Medicine (PubMed), and Web of Science. The descriptors in Portuguese and English were used: Varicose Ulcer AND Hyperbaric Oxygen Therapy. The association of descriptors enabled the selection of forty (40) articles without including a time limit, five (05) articles were included in the review. Hyperbaric oxygen therapy showed a high potential healing effect on chronic venous wounds. The evinced effects were: improvement of tissue hypoxia, increased perfusion, reduction of edema, decrease in the regulation of inflammatory cytokines, proliferation of fibroblasts, collagen production and angiogenesis. The review showed efficacy in the treatment of complex venous ulcers with hyperbaric oxygenation, as there was a reduction in pain scores, an increase in the healing and curing process, however, it was not possible to observe effects in non-complex ulcers.
\end{abstract}

Keywords: Varicose ulcer, Oxygen inhalation therapy, Wound healing.

${ }_{1}$ Centro Universitário UniJorge, Salvador - BH. *E-mail: renata.s.schulz@gmail.com

2 Universidade Federal da Bahia, Salvador - BH. 
Resumen: Este artículo pretende verificar en la literatura los beneficios de la oxigenoterapia hiperbárica en personas con úlceras venosas. Esta es una revisión integral realizada en las bases de datos electrónicas Biblioteca Virtual de Salud (BVS) National Library de Medicine (PubMed), y Web of Science. Se utilizaron los descriptores en portugués e inglés: úlcera varicosa y Terapia por Inhalación de Oxígeno. La asociación de descriptores permitió la selección de cuarenta (40) artículos sin incluir un límite de tiempo, se incluyeron cinco (05) artículos en la revisión. La oxigenoterapia hiperbárica tuvo un alto potencial de curación en las heridas venosas crónicas. Los efectos demostrados fueron: mejoría de la hipoxia tisular, aumento de la perfusión, reducción del edema, disminución de la regulación de las citocinas inflamatorias, proliferación de fibroblastos, producción de colágeno y angiogénesis. La revisión demostró eficacia en el tratamiento de úlceras venosas compleja con oxigenación hiperbárica, ya que hubo una reducción en las puntuaciones de dolor, un aumento en el proceso de disminución de área y curación, no fue posible observar efectos en úlceras no complejas.

Palabras-clave: Úlcera varicosa, Terapia por inhalación de oxígeno, Cicatrización de heridas.

\section{INTRODUÇÃO}

As úlceras venosas são crônicas e envolvem processos patológicos macroscópicos e microscópios do sistema venoso, há alterações de fatores genéticos, hipertensão venosa, inflamação crônica dos vasos, extravasamento de leucócitos e de citocinas pró-inflamatórias, sobrecarga de ferro, tensões na derme que favorecem taxas de recorrência das lesões após 5 anos de cura, essas taxas são maior que $58 \%$ (CRAWFORD JM, et al., 2017)

Estima-se, no Reino Unido, que o custo para o tratamento das úlceras venosas seja de 300 a 450 milhões de libras por ano, e que as enfermeiras dedicam $25 \%$ a $50 \%$ do seu tempo ao cuidado de pessoas com úlceras venosas (KRANKE P, et al., 2004). É notório que a incidência aumenta com o avançar da idade, principalmente nas pessoas que possuem mais de 80 anos a proporção chega a ser de 20 por 1.000 habitantes (KRANKE P, et al., 2004). Desse modo, as pessoas com mais de 80 anos têm maior probabilidade de desencadear úlcera venosa, causada por obstrução devido a pressão venosa elevada.

Para o tratamento da úlcera venosa é importante ações interdisciplinares, a atuação das especialidades de enfermagem, medicina, fisioterapia, nutrição, psicologia, serviço social, educação física precisa ser integrada, para que se efetive uma melhor atuação desses profissionais em direção à cura e melhor qualidade de vida durante o tratamento (JOAQUIM FL, et al., 2018).

As úlceras venosas comprometem a saúde da pessoa acometida de forma física, emocional e social (JOAQUIM FL, et al., 2018). Para que haja êxito na qualidade de vida dos pacientes, é importante proporcionar atividades que amenizem as tensões emocionais, que permitam o bem-estar e a independência das pessoas em processo de cicatrização (JOAQUIM FL, et al., 2018). Além disso, é necessário reavaliar medicamentos utilizados, garantir atividade física adequada, (LENTSCK MH, et al., 2018) alterar hábitos alimentares (MENDES CA e MOTTA JB, 2018), pois estes são fatores que auxiliam no processo da melhora cicatricial.

Dentre os inúmeros tratamentos voltados a úlcera venosa a oxigenoterapia hiperbárica é uma modalidade terapêutica que consiste na administração de oxigênio puro, pela via respiratória, a pessoa em tratamento é colocada em uma câmara hiperbárica, na qual são aplicadas pressões superiores à pressão atmosférica (ANDRADE SM e SANTOS ICRV, 2016). A tensão de oxigênio tecidual é um dos mais importantes fatores de cicatrização das feridas já que o oxigênio atua como substrato para enzimas que atuam no processo cicatricial, favorece a epitelização, a síntese e depósito de colágeno, a angiogênese, a resistência e combate à infecção (RODRIGUES JUNIOR M e MARRA AR, 2015).

Vale destacar que no Brasil a oxigenoterapia hiperbárica é um tratamento realizado por indicação médica, seu custo é elevado cerca de 300 reais a sessão e todo o tratamento pode custar entre 1 mil a 18 mil reais (HCUFMG/NATS, 2014). É indicada para lesões graves e casos complexos e não está indicada para úlceras venosas com resposta satisfatória ao tratamento habitual. A terapia está indicada para recuperação de tecidos em sofrimento; condições clínicas em que seja o único tratamento; falha de resposta aos tratamentos 
habituais; lesões com necessidade de desbridamento cirúrgico; piora rápida com risco de óbito; lesões em áreas nobres; lesões refratárias e recidivas frequentes (OLIVEIRA R, et al., 2017).

A oxigenoterapia hiperbárica se apresenta como alternativa adjuvante no tratamento de úlcera venosa para os casos sem resposta ao tratamento. Ela resulta na melhora da qualidade de vida dos pacientes tratados, mas também é considerável a redução do tempo e do gasto com internações hospitalares muitas vezes prolongadas, procedimentos intervencionistas, uso de antibioticoterapia, além de curativos diários (RODRIGUES JUNIOR M e MARRA AR, 2015).

Ao considerar as repercussões socioeconômicas e psicossociais que podem ser vividas pelas pessoas com úlcera venosa incluindo os custos elevados com os cuidados/tratamento e a demora da cicatrização nesse tipo de ferimento, torna-se importante que os cuidados prestados sejam eficazes, a fim de gerar benefícios adequados que favoreça e acelere o processo de cicatrização (JOAQUIM FL, et al., 2018).

A motivação pela escolha desse tema teve origem no sexto semestre durante aulas práticas vivenciadas em um Centro de Saúde na cidade de Salvador - BH. Durante a realização dos atendimentos e ao analisar as evoluções dos ferimentos por meio de prontuários era notório que a cicatrização das úlceras venosas era lenta, ao utilizar apenas o curativo convencional como terapia. Foi possível perceber o crescente número de pessoas acometidas pela patologia que procuram atendimento e as alterações nas atividades de vida diárias dessas pessoas. Diante disso, surgiu como pergunta de pesquisa: Quais os benefícios que a terapia hiperbárica possui em relação ao tratamento da úlcera venosa e como objetivo traçou-se: Verificar, na literatura, os benefícios da oxigenoterapia hiperbárica em pessoas com úlcera venosa.

\section{MÉTODOS}

O estudo faz parte de uma revisão integrativa da literatura que é elaborada por meio de uma síntese pautada que pode abranger diferentes tópicos, é uma metodologia utilizada como primeiro passo para a construção do conhecimento científico (BOTELHO LLR, et al., 2011). Esse método de estudo é desenvolvido de acordo as seguintes etapas: identificação do tema e definição da questão norteadora; estabelecimento dos critérios para a seleção da amostra; definição dos dados a serem extraídos dos estudos selecionados e sua categorização; avaliação dos estudos incluídos na revisão integrativa; e interpretação dos resultados e conclusão da revisão.

A coleta dos artigos para essa revisão ocorreu em março de 2020. Para localização dos artigos foram utilizadas as bases de dados onde foram identificados inicialmente um total de 40 artigos distribuídos nas seguintes bases: Biblioteca Virtual de Saúde (BVS) (8), National Library of Medicine (PubMed) (29), e Web of Science (3). O processo de seleção dos artigos teve como critério a associação dos descritores em inglês e português combinados pelo operador booleano AND desse modo utilizou-se na busca: Úlcera Varicosa AND Oxigenoterapia.

Os critérios de inclusão dos artigos foram: artigos gratuitos, disponíveis na íntegra, sem recorte temporal, indexação nas bases de dados especificadas anteriormente, escritos em inglês e em português. Foram excluídos os artigos repetidos (8) e os que não apresentaram o tema de úlcera venosa para oxigenoterapia hiperbárica (27).

Inicialmente, houve a leitura do título da publicação, seguida pela leitura criteriosa do resumo dos artigos, realizada por quatro revisoras de maneira individual, para verificar a adequação dos critérios de seleção. Em seguida foi efetuada a leitura dos textos, na íntegra, a fim de verificar a adequação aos critérios de inclusão e exclusão. Em outro momento, houve uma discussão sobre a adequação dos artigos selecionados aos critérios de inclusão e exclusão para seleção, por cada revisora, a fim de alcançar um consenso.

\section{RESULTADOS}

Foram encontrados 40 artigos após aplicar os critérios de inclusão restaram 13, 08 estiveram repetidos, e 5 artigos foram selecionados para fazer parte deste estudo, conforme demonstrado na Figura 1. Foram

REAEnf/EJNC | Vol. 5 | e4921 | DOI: https://doi.org/10.25248/REAenf.e4921.2020 Página 3 de 8 
encontrados artigos publicados nos anos de $1970(n=1), 2009(n=1), 2016(n=1)$ e $2018(n=2)$ n. A seleção encontrou trabalhos originais $(n=3)$, de análise documental $(n=1)$ e revisão sistemática $(n=1)$. Os estudos foram desenvolvidos no Brasil, na Austrália, na Polônia e na Inglaterra.

Figura 1 - Seleção dos artigos para a revisão. Março de 2019.

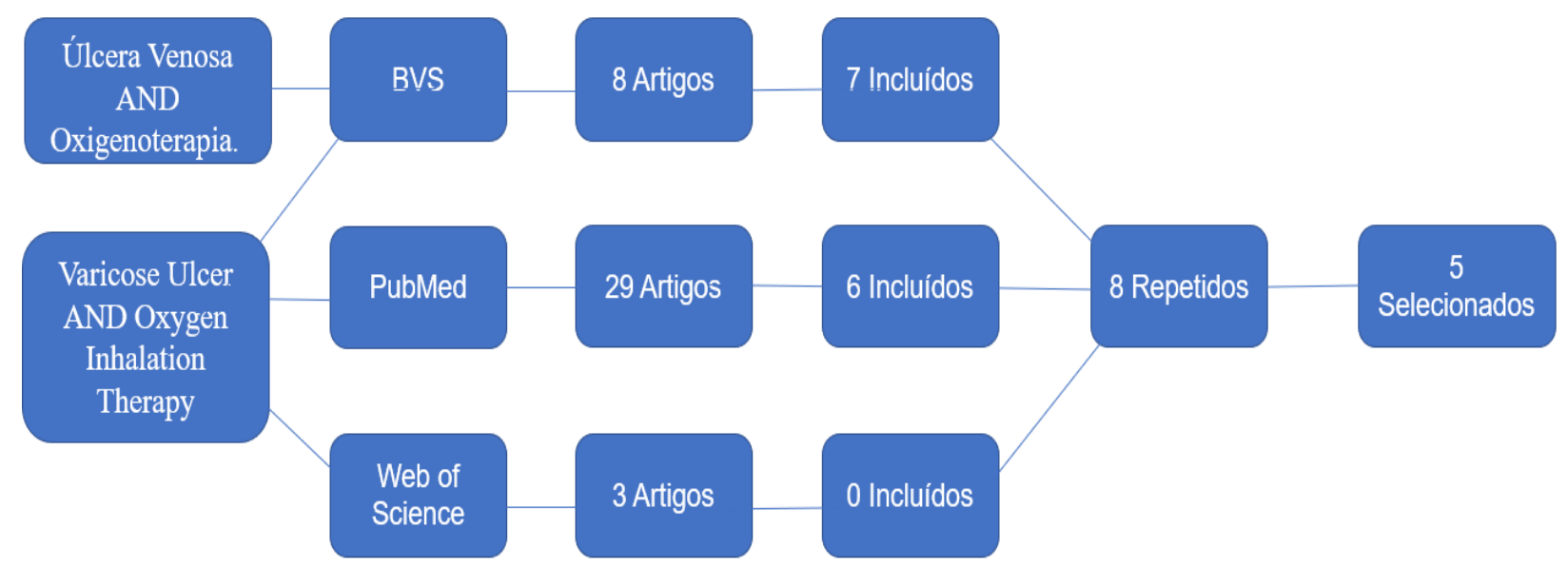

Fonte: Lima LO, et al., 2020.

Os principais benefícios encontrados nos artigos desta revisão integrativa foram: Menor número de sessões da terapia hiperbárica; melhora da cicatrização, redução da lesão e cura; melhora do exsudato e epitelização; fechamento precoce da ferida utilizando enxertos e OHB; melhora da qualidade de vida e da dor; melhora da infecção (ANDRADE SM e SANTOS ICRV, 2016; KRANKE P, et al., 2004; THISTLEHWAITE KR, et al., 2018; GLIK J, et al., 2019 e BASS BH, 1970). No Quadro 1 tem-se um quadro com descrição da quantidade dos benefícios. Já os artigos incluídos na revisão estão representados no Quadro 2.

Quadro 1 - Quantidade de trabalhos que registram os tipos benefícios da oxigenoterapia hiperbárica na úlcera venosa

\begin{tabular}{|c|c|}
\hline Tipos de benefícios & $\begin{array}{c}\text { Quantidade dos } \\
\text { trabalhos que } \\
\text { indicam os tipos de } \\
\text { benefícios }\end{array}$ \\
\hline Melhora da cicatrização & 1 \\
\hline Redução da lesão & 5 \\
\hline Cura & 5 \\
\hline Melhora do exsudato e epitelização & 1 \\
\hline Fechamento precoce da ferida utilizando enxertos e OHB & 1 \\
\hline Melhora da qualidade de vida e da dor & 1 \\
\hline Melhora da infecção & \\
\hline
\end{tabular}

Fonte: Lima LO, et al., 2020. 
Revista Eletrônica Acervo Enfermagem / Electronic Journal Nursing Collection | ISSN 2674-7189

Quadro 2 - Descrição dos artigos incluídos na revisão integrativa incluindo: Ano, Autores, Título, Delineamento do estudo e Desfechos.

\begin{tabular}{|c|c|c|c|}
\hline $\begin{array}{l}\text { Ano/País } \\
\text { Autores }\end{array}$ & Título & Delineamento do estudo & Desfechos \\
\hline $\begin{array}{l}\text { 1970/ Inglaterra/ } \\
\text { BASS BH }\end{array}$ & $\begin{array}{l}\text { The treatment of varicose leg } \\
\text { ulcers by hyperbaric oxygen. }\end{array}$ & $\begin{array}{l}\text { Estudo experimental com } 19 \text { pessoas } \\
\text { com úlcera venosa. }\end{array}$ & $\begin{array}{l}\text { As úlceras varicosas cicatrizam rapidamente após uma exposição média de 1a } 7 \text { horas. } \\
\text { Dezessete dos pacientes receberam alta completa de cura. A sepse atrasou o processo de } \\
\text { cicatricial. Os pacientes foram expostos durante cinco dias por semana, a duas horas de } \\
\text { sessões diárias, com a supervisão de uma enfermeira. Houve coleta de exsudação das } \\
\text { úlceras, com cotonetes, para análise do efeito do oxigênio hiperbárico nos organismos } \\
\text { infectantes. }\end{array}$ \\
\hline $\begin{array}{l}\text { 2009/Alemanha/ } \\
\text { KRANKE P, et al. }\end{array}$ & $\begin{array}{l}\text { Hyperbaric oxygen therapy } \\
\text { for chronic wounds. }\end{array}$ & $\begin{array}{l}\text { Trata-se de uma revisão sistemática } \\
\text { com } 5 \text { ensaios clínicos sobre a } \\
\text { terapia hiperbárica. }\end{array}$ & $\begin{array}{l}\text { A revisão apresenta que houve benefício com a utilização da terapia hiperbárica. A revisão } \\
\text { apresenta um dos ensaios com } 16 \text { pacientes de úlcera venosa. Houve redução da dimensão } \\
\text { da ferida após dezoito semanas, seis semanas de tratamento. }\end{array}$ \\
\hline $\begin{array}{l}\text { 2016/ Brasil/ } \\
\text { ANDRADE SM e } \\
\text { SANTOS ICRV }\end{array}$ & $\begin{array}{l}\text { Oxigenoterapia hiperbárica } \\
\text { para tratamento de feridas. }\end{array}$ & $\begin{array}{l}\text { Trata-se de um estudo transversal e } \\
\text { documental. Conduzido em um } \\
\text { Centro Hiperbárico referência na } \\
\text { cidade do Salvador, Bahia. }\end{array}$ & $\begin{array}{c}\text { Foram analisados } 200 \text { prontuários dos pacientes com feridas que realizaram tratamento com } \\
\text { terapia hiperbárica. Nestes foram verificadas que as mais frequentes foram: úlcera venosa } \\
(21 \%) \text { e lesão traumática }(21 \%) \text {, seguidas por pé diabético }(17 \%) \text {. Os pacientes com feridas } \\
\text { crônicas realizaram um menor número de sessões }(61,1 \%) \text { e tiveram suas feridas cicatrizadas } \\
\text { ou reduzidas }(62,0 \%) \text { quando comparados com aqueles com feridas agudas. Também foi } \\
\text { possível identificar a lacuna ainda existente quanto a atuação da enfermagem na } \\
\text { oxigenoterapia hiperbárica, apontando para necessidade de novas pesquisas sobre a temática } \\
\text { voltadas para o ensino em saúde. }\end{array}$ \\
\hline $\begin{array}{l}\text { 2018/ Austrália/ } \\
\text { THISTLEHWAITE } \\
\quad \text { KR, et al. }\end{array}$ & $\begin{array}{l}\text { The effectiveness of } \\
\text { hyperbaric oxygen therapy } \\
\text { for healing chronic venous } \\
\text { leg ulcers: A randomized, } \\
\text { double-blind, placebo- } \\
\text { controlled trial }\end{array}$ & $\begin{array}{l}\text { Estudo randomizado, duplo-cego, } \\
\text { controlado por placebo. }\end{array}$ & $\begin{array}{l}\text { Estudo desenvolvido na Austrália, participaram do estudo } 58 \text { pacientes, sendo } 29 \text { em cada } \\
\text { grupo. A principal descoberta positiva do estudo foi que em } 12 \text { semanas de tratamento, após o } \\
\text { início da intervenção, o grupo com terapia hiperbárica apresentou uma redução na área da } \\
\text { úlcera significativamente maior de } 95 \% \text {, em comparação com } 54 \% \text { no grupo placebo. } \\
\text { O grupo com terapia hiperbárica recebeu } 30 \text { sessões de tratamento com oxigenoterapia } \\
\text { hiperbárica durante } 120 \text { min/ } 5 \text { vezes por semana. Os participantes randomizados para o } \\
\text { grupo placebo receberam ar pressurizado inicialmente com uma pressão elevada e, logo em } \\
\text { seguida a pressão circulou de forma ascendente e descendente, por cerca de } 8 \text { minutos, } \\
\text { antes de estabelecer a pressão absoluta. }\end{array}$ \\
\hline $\begin{array}{l}\text { 2019/Polônia / GLIK } \\
\text { J, et al. }\end{array}$ & $\begin{array}{l}\text { Thermal imaging and } \\
\text { planimetry evaluation of the } \\
\text { results of chronic wounds } \\
\text { treatment with hyperbaric } \\
\text { oxygen therapy }\end{array}$ & $\begin{array}{l}\text { Estudo longitudinal acompanhado por } \\
\qquad 3 \text { anos. }\end{array}$ & $\begin{array}{l}\text { Durante um período de três anos, foram coletadas } 284 \text { medidas de planimetria por } \\
\text { computador digital de } 142 \text { pacientes tratados por úlceras nas pernas causadas por } \\
\text { insuficiência venosa crônica e úlceras por síndrome do pé diabético na Polônia. Cada paciente } \\
\text { realizou } 30 \text { sessões de terapia hiperbárica usando uma câmara Hauxmultiplace a uma } \\
\text { pressão de } 2,5 \text { atmosferas absolutas. Houve redução no perímetro da ferida, bem como maior } \\
\text { progresso na cicatrização, permitindo o fechamento mais precoce da ferida por pele de } \\
\text { espessura intermediária de enxertos. Para obter dados objetivos sobre o processo de } \\
\text { cicatrização, foram utilizadas planimetria digital (medição computadorizada) que permitiu } \\
\text { medir feridas em imagens digitais traçando seus contornos usando um computador } \\
\text { especializado Software e imagens térmicas utilizada para monitorar a distribuição da } \\
\text { temperatura da pele. }\end{array}$ \\
\hline
\end{tabular}

Fonte: Lima LO, et al., 2020.

REAEnf/EJNC | Vol. 5 | e4921 | DOI: https://doi.org/10.25248/REAenf.e4921.2020 Página 5 de 8 


\section{DISCUSSÃO}

Observa-se pela revisão que a terapia hiperbárica tem efeito de alto potencial para feridas que são difíceis de obter a cura, como nas feridas venosas. (THISTLEHWAITE KR, et al., 2018). A terapia hiperbárica pode reduzir o número de amputações em pessoas com patologias diferentes (ABIDIA A, et al., 2003). Além disso há redução do exsudato ocorre visivelmente após as primeiras 2 horas de exposição, e a nova pele que cresce na periferia, epitelização da úlcera, tem uma cor rosa saudável (BASS BH, 1970).

$\mathrm{O}$ tratamento foi mais rápido e eficaz os pacientes puderam retornar às suas vidas normais após a terapêutica o que favoreceu a cicatrização das úlceras (BASS BH, 1970). Por se ser um tratamento relativamente rápido, o custo-benefício é relevante, pois em técnicas de curativos tradicionais o tempo que uma ferida leva para cicatrizar é muito maior. Em muitos tratamentos de úlceras venosas é possível identificar o sofrimento dos pacientes na realização do curativo, não só devido a dor, mas também com a demora da cicatrização, com a utilização da terapia hiperbárica é notório que esses dois aspectos sejam mais toleráveis (ANDRADE SM e SANTOS ICRV, 2016).

Os efeitos demonstrados experimentalmente pela oxigenoterapia foram: melhora da hipóxia tecidual, aumento da perfusão, redução do edema, queda na regulação das citocinas inflamatórias, proliferação de fibroblastos, produção de colágeno e angiogênese (THISTLEHWAITE KR, et al., 2018). A oxigenoterapia também é indicada para a erradicação de infecções dos tecidos moles e ósseos, difíceis de tratar por mecanismos que incluem destruição de microrganismos, melhorando a função de leucócitos e macrófagos (ANDRADE SM e SANTOS ICRV, 2016).

Em relação aos efeitos colaterais identificou-se poucos e estiveram relacionados à variação da pressão e/ou toxicidade do oxigênio, sendo os principais o barotrauma auditivo, desconforto em seios da face, comprometimento do cérebro, pulmões e alterações visuais transitórias (KRANKE P, et al., 2004).

Vale lembrar que a terapia hiperbárica possui como contraindicações absolutas 0 uso de drogas quimioterápicas, diagnóstico de pneumotórax não tratado e gravidez (KRANKE P, et al., 2004). Porém, quando indicada pode beneficiar a melhora a cicatrização, o edema, o exsudato, favorecer o processo de cura, pois permite melhora da qualidade de vida das pessoas com úlcera venosa.

Vale destacar que a razão da utilização da oxigenoterapia é que muitas feridas levam à hipóxia, entretanto as pessoas não podem permanecer mais de duas (02) horas na câmara, pois uma elevada pressão pode prejudicar os pulmões, o chamado barotrauma (ANDRADE SM e SANTOS ICRV, 2016).

O tempo de permanência na câmara hiperbárica variou entre os estudos. Verificou-se a qualidade de vida e as medidas de dor, os participantes receberam tratamento de até 120 minutos por dia na câmara hiperbárica, cinco (05) dias por semana e o grupo placebo recebeu ar pressurizado (THISTLEHWAITE KR, et al., 2018). Houve como fator positivo redução média da úlcera maior que $95 \%$ em comparação com $54 \%$ no grupo placebo (THISTLEHWAITE KR, et al., 2018).

Já os pacientes receberam trinta 30 sessões de oxigenoterapia, realizadas a cada 24 horas, cinco (05) vezes por semana durante 90 minutos. Observou-se que $16,32 \%$ dos pacientes obtiveram cura e, $83 \%$, dos pacientes conseguiram uma redução significativa da ferida para os tratamentos com enxerto de pele (GLIK J, et al., 2019). No estudo controlado por placebo para tratamento com oxigenoterapia os participantes receberam a terapêutica durante 12 semanas, sendo avaliados semanalmente. Na quarta semana, observouse redução de $50 \%$ da área das feridas e depois cura (THISTLEHWAITE KR, et al., 2018).

Os escores de dor diminuíram para os dois grupos (placebo e grupo de tratamento), porém com média maior e favorável de diminuição da dor para o grupo com o tratamento de oxigenoterapia, este é um fator que favorece na qualidade de vida dos pacientes (THISTLEHWAITE KR, et al., 2018),

Observou-se que ao incluir acompanhamentos digitais para a avaliação da comprovação da eficácia da terapia hiperbárica, a avaliação feita por meio de imagens térmicas e planimetria digital por computador permitiram medir as feridas em imagens digitais ao traçar seus contornos usando computador especializado e software (GLIK J, et al., 2019). O sistema digital fornece a gravação, foram encontrados erros de medição 
ao utilizar outras técnicas, como régua e filme transparente, com isso as imagens térmicas e de planimetria podem ser utilizadas para avaliar a evolução da cicatrização da área.

Assim, a medição com a planimetria possibilitou a equipe multidisciplinar uma avaliação precisa. Desta forma, influenciou diretamente na escolha dos métodos de tratamento das úlceras e favoreceu a diminuir dos custos com a terapia. As imagens térmicas consistem na distribuição da temperatura na superfície da pele que foram monitoradas com o uso de uma câmera Thermovision E60, desse modo permitiu visualizar o metabolismo e atividade tecidual antes e após as sessões de oxigenoterapia (GLIK J, et al., 2019). Mais de $30 \%$ das úlceras de perna venosa não responderam aos cuidados padrões e necessitam de terapia combinada (KRANKE P, et al., 2004).

É importante ressaltar que precisa haver uma seleção dos pacientes com úlcera venosa para a terapêutica da oxigenoterapia hiperbárica, para favorecer a cicatrização da úlcera venosa. Os participantes precisam ter mais de 18 anos, diagnóstico clínico de úlcera venosa, levando em consideração seu índice de pressão braquial (THISTLEHWAITE KR, et al., 2018). Os artigos encontrados indicam que a oxigenoterapia deve ser utilizada em feridas que não respondam aos cuidados normais, ou seja, nas feridas crônicas, para que assim, haja evolução em poucas sessões o que pode justificar a seleção de 05 artigos apenas.

O tratamento pela sua complexidade exige também o seguimento de cuidados direcionados, que favoreçam a cicatrização; que se constituem de: dieta adequada, repouso, elevar membros inferiores, limpeza adequada da ferida (que deve reduzir as chances de traumas mecânicos e químicos no leito da ferida e manter a temperatura local em torno de $37^{\circ} \mathrm{C}$, em relação a ferida os cuidados envolvem: remoção de fragmentos de tecido necrótico, debris, resíduos da cobertura anterior), avaliar o excesso de exsudato, a diminuição do número de microrganismos na lesão, o uso de meias de compressão após a cura da ferida, aplicar produto adequado de acordo com o tecido existente no leito da ferida (ABBADE FPL e LASTÓRIA S, 2006). Esses cuidados são utilizados para suprir as necessidades afetadas em consequência da úlcera venosa, da ruptura do tecido, da insuficiência no bombeamento do sangue e do déficit de oxigenação no leito da ferida (ABIDIA A, et al., 2003).

São muito utilizadas, no Brasil, as terapias compressivas, por favorecer a circulação de fluidos no interstício levando-os aos compartimentos linfáticos e vascular, aumentando a atividade da panturrilha (MENDES CA e MOTTA JB, 2018). O ideal nos casos da úlcera com necrose é realizar o desbridamento antes da utilização de meias ou dispositivos compressivos. Porém, não há, ainda, um tratamento que possa ser definido como exclusivo e efetivo para todos os casos de úlceras crônicas, independente da etiologia da úlcera deve ser tratada a doença de base, não somente a lesão para que o quadro tenha uma evolução positiva (JOAQUIM FL, et al., 2018).

As úlceras venosas são consideradas um problema de saúde pública de difícil cicatrização que afeta a qualidade de vida. Os centros de terapia hiperbárica auxiliam no tratamento de lesões crônicas e devem atuar de modo associado à prescrição de cuidados, porém, não são acessíveis à população em diversas regiões e instituições brasileiras. Desta forma, é possível evidenciar que a oxigenoterapia é uma terapia alternativa de alto potencial para o tratamento de úlcera venosa complexa, visto que, além de proporcionar melhora na qualidade de vida dos portadores, favorece a cicatrização o que reduz gastos com internações hospitalares e com curativos diários. Como limitação deste estudo, aponta-se uma referência que embora significativa data da década de 70, embora a oxigenoterapia seja um tratamento de alto potencial, os artigos excluídos envolveram outras patologias, como as lesões em pé diabético e diversas outras patologias. Assim, sugerese novas pesquisas de caráter longitudinal com corte temporal atualizado, que demonstrem melhor efetividade da oxigenoterapia, em relação ao tratamento convencional da úlcera venosa

\section{CONSIDERAÇÕES FINAIS}

A revisão integrativa permitiu descrever os benefícios da oxigenoterapia hiperbárica no tratamento de úlcera venosa complexa, não foi possível avaliar o benefício em úlceras venosas comuns. Os artigos encontrados conseguiram evidenciar como foi possível alcançar a cicatrização ou redução da ferida com melhora nos escores de dor em comparação com seus placebos em úlceras venosas que não responderam

REAEnf/EJNC | Vol. 5 | e4921 | DOI: https://doi.org/10.25248/REAenf.e4921.2020 Página 7 de 8 
ao tratamento convencional. Porém, não se pode definir um único tratamento como exclusivo para todos os casos de úlceras venosas crônicas, já que é necessário tratar também a doença de base, para que o quadro tenha melhor evolução.

\section{REFERÊNCIAS}

1. ABBADE FPL, LASTÓRIA S. Management of patients with venous leg ulcer. Anais Brasileiros de Dermatologia, 2006; 81(6): 509-522.

2. ABIDIA A, et al. The role of hyperbaric oxygen therapy in ischaemic diabetic lower extremity ulcers: A double-blind randomised-controlled trial. European Journal of Vascular and Endovascular Surgery, 2003; 25(6): 513-518.

3. ANDRADE SM, SANTOS ICRV. Hyperbaric oxygen therapy for wound care. RevistaGaúcha de Enfermagem, 2016; 37(2).

4. BASS BH. The treatment of varicose leg ulcers by hyperbaric oxygen. Postgraduate Medical Journal, 1970; 46(537): 407-408.

5. BOTELHO LLR, et al. O método da revisão integrativa nos estudos organizacionais. RevistaEletrônicaGestão e Sociedade, 2011; 5(11): 121-136.

6. CRAWFORD JM, et al. Pathophysiology of venous ulceration. Doença do linfático venoso J Vasc Surg, 2017; 5(4): 596-605.

7. GLIK J, et al. Thermal imaging and planimetry evaluation of the results of chronic wounds treatment with hyperbaric oxygen therapy. Advances in Clinical and Experimental Medicine, 2019; 28(2): 229-236.

8. KRANKE P, et al. Hyperbaric oxygen therapy for chronic wounds. Cochrane Database of Systematic Reviews, 2004; 3: 1-40.

9. LENTSCK MH, et al. Quality of life related to clinical aspects in people with chronic wound. Revista da Escola de Enfermagem da USP, 2018; 52.

10. JOAQUIM FL, et al. Impact of venous ulcers on patients' quality of life: an integrative review. Revista Brasileira de Enfermagem, 2018; 71(4): 2137-2146.

11. NÚCLEO DE AVALIAÇÃO EM TECNOLOGIA EM SAÚDE (NATS). HOSPITAL DAS CLÍNICAS UNIVERSIDADE FEDERAL DE MINAS GERAIS (HCUFMG). Nota técnica 34/2014. Oxigenoterapia hiperbárica para paciente com úlcera varicosa e diabetes. Minas Gerais, 2014.

12. MENDES CA, MOTTA JB. Tratamento das úlceras crônicas de membros inferiores: estado da arte e perspectivas futuras. Hegemonia - Revista Eletrônica do Programa de Mestrado em Direitos Humanos, Cidadania e Violência/Ciência Política do Centro Universitário Unieuro, 2018; 12(25): 137-149.

13. OLIVEIRA R, et al. Protocolo de oxigenoterapia hiperbárica. Segundo orientação da Sociedade Brasileira de Medicina Hiperbárica e Resolução Conselho Federal de Medicina n.ำ 1.457/1995 e Comissão Nacional de Incorporação de Tecnologias do SUS. Governo do Estado do Espírito Santo Secretaria da Saúde, 2017.

14. RODRIGUES JUNIOR M, MARRA AR. Quando indicar a oxigenoterapia hiperbárica? Revista da Associação Médica Brasileira, 2004; 50(3): 229-251.

15. THISTLEHWAITE KR, et al. The effectiveness of hyperbaric oxygen therapy for healing chronic venous leg ulcers: $A$ randomised, double blind, placebo-controlled trial. WoundRepair and Regeneration, 2018; 26(4): 324-331. 